\title{
Oxidative Phosphorylation Promotes Primary Melanoma Invasion
}

Amel Salhi, ${ }^{*}$ Alexander C. Jordan, ${ }^{*}$ Irineu I. Bochaca, ${ }^{*}$ Allison Izsak, ${ }^{*}$ Farbod Darvishian, ${ }^{\dagger}$ Yariv Houvras, ${ }^{\ddagger}$ Keith M. Giles, ${ }^{*}$ and Iman Osman*

From the Ronald O. Perelman Department of Dermatology, * and the Department of Pathology, ${ }^{\dagger}$ New York University School of Medicine, New York; and the Department of Surgery, ${ }^{\ddagger}$ Weill Cornell Medical College, New York, New York

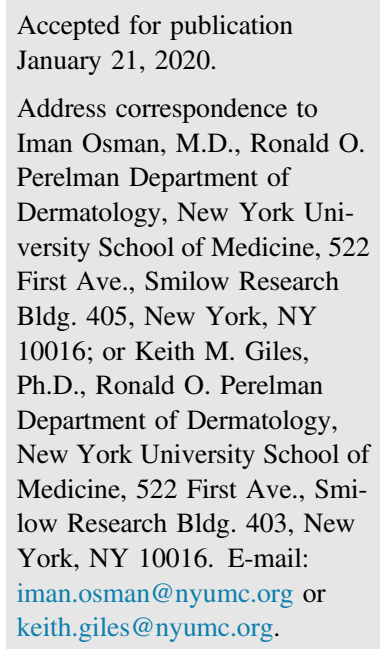

\begin{abstract}
Dermal invasion is a hallmark of malignant melanoma. Although the molecular alterations that drive the progression of primary melanoma to metastatic disease have been studied extensively, the early progression of noninvasive primary melanoma to an invasive state is poorly understood. To elucidate the mechanisms underlying the transition from radial to vertical growth, the first step in melanoma invasion, we developed a zebrafish melanoma model in which constitutive activation of ribosomal protein S6 kinase A1 drives tumor invasion. Transcriptomic analysis of ribosomal protein S6 kinase A1activated tumors identified metabolic changes, including up-regulation of genes associated with oxidative phosphorylation. Vertical growth phase human melanoma cells show higher oxygen consumption and preferential utilization of glutamine compared to radial growth phase melanoma cells. Peroxisome proliferator activated receptor $\gamma$ coactivator (PGC)-1 $\alpha$, has been proposed as a master regulator of tumor oxidative phosphorylation. In human primary melanoma specimens, $\mathrm{PGC} 1 \alpha$ protein expression was found to be positively associated with increased tumor thickness and expression of the proliferative marker Ki-67 and the reactive oxygen species scavenger receptor class A member 3. PGC $1 \alpha$ depletion modulated cellular processes associated with primary melanoma growth and invasion, including oxidative stress. These results support a role for $\mathrm{PGC} 1 \alpha$ in mediating glutamine-driven oxidative phosphorylation to facilitate the invasive growth of primary melanoma. (Am J Pathol 2020, 190: 1108-1117; https://doi.org/10.1016/j.ajpath.2020.01.012)
\end{abstract}

The molecular mechanisms underlying melanoma metastasis have been studied extensively; however, the processes that control the early progression of primary melanoma are not well understood. During the radial growth phase (RGP), primary melanoma cells are confined to the epidermis. As the earliest step of progression, a melanoma transitions to a vertical growth phase (VGP), in which tumor cells invade the dermis and develop the capacity for metastasis. ${ }^{1}$ This invasive behavior is a hallmark of melanoma and is thought to reflect its neural crest origin. ${ }^{2}$ Some studies have identified molecular alterations that contribute to primary melanoma invasion. For example, deregulation of transcription factors, such as cyclic AMP-responsive element binding protein, leads to changes in expression or function of adhesion molecules, matrix-degrading enzymes, and survival factors that promote melanoma cell invasion. $^{3}$ Oncogenic activation of BRAF V600E in human melanocytes results in up-regulation of genes associated with growth, motility, inflammation, and extracellular matrix degradation. ${ }^{4}$ Multiple miRNAs, including miR-382 and miR-516b, have been shown to alter the expression of genes that are crucial for melanoma invasion and progression. ${ }^{5}$ However, to date no studies have reported a role for altered metabolism in the early progression of primary melanoma.

The importance of metabolic reprogramming in cancer initiation and progression is recognized, ${ }^{6,7}$ and melanoma

Supported by NIH National Cancer Institute, New York University (NYU) Cancer Institute Cancer Center Support grant P30CA016087, NYU Melanoma Specialized Program of Research Excellence (SPORE) grant P50CA016087 (I.O.), Goldberg Charitable Trust, and the Wings for Things Foundation.

Disclosures: None declared. 
cells are able to utilize and regulate multiple metabolic pathways to sustain their proliferation, invasion, and metastasis, including glycolysis, oxidative phosphorylation (OXPHOS), tricarboxylic acid cycle, and amino acid and lipid metabolism. ${ }^{8}$ Induction of the transcription coactivator peroxisome proliferator-activated receptor $\gamma$ coactivator (PGC)-1 $\alpha$ (PPARGC1A) in a subset of metastatic melanomas by the melanocyte lineage factor microphthalmiaassociated transcription factor (MITF) has been shown to promote mitochondrial capacity, OXPHOS, and resistance to oxidative stress and mitogen-activated protein kinase pathway inhibitors, ${ }^{9-11}$ but its relevance in primary melanoma remains to be established. Here, we tested the hypothesis that an altered metabolic state promotes invasive growth of primary melanoma, using an integrated approach that utilized a genetic zebrafish model of invasive melanoma, as well as human melanoma cell lines and patient tumor specimens.

\section{Materials and Methods}

\section{Zebrafish Melanoma Model}

To develop a zebrafish model of invasive melanoma, MiniCoopR vectors were generated as described. ${ }^{12}$ An Nterminal myristoylation sequence (Myr-RSK1) was added to generate constitutively active human ribosomal protein S6 kinase A1 (RSK1; RPS6KA1). ${ }^{13}$ For melanoma assays, microinjection of zebrafish embryos was performed from an in-cross of $\operatorname{Tg}[$ mitfa:BRAF(V600E);p53(M214K/ M214K);nacre]. Melanocyte-rescued animals were raised to adulthood and observed weekly for melanoma onset as previously described. ${ }^{14}$ Tumor invasion was assessed by histology, and melanoma tissue was used for RNA extraction for RNA sequencing (RNA-Seq).

\section{Melanoma Cell Culture and RNA Interference}

Primary melanoma cell lines (WM35, WM278, WM115, and WM3248) were obtained from the Wistar Institute (Philadelphia, PA), used within 20 passages after resuscitation, and cultured in MCDB153/L15 medium (4:1 v/v) supplemented with $2 \%$ fetal bovine serum, $5 \mathrm{mg} / \mathrm{mL}$ insulin, $15 \mathrm{mg} / \mathrm{mL}$ bovine pituitary extract, $1.68 \mathrm{mmol} / \mathrm{L}$ calcium chloride, $5 \mathrm{ng} / \mathrm{mL}$ epidermal growth factor, and $1 \%$ penicillin/streptomycin.

To knock down PGC1 $\alpha$ expression, siRNAs were purchased from Qiagen (Germantown, MD): PPARGC1A (PGC1 $\alpha$; catalog number SI02639833; AllStars negative control: catalog number 102780). WM3248 melanoma cells were transfected with siRNA against PGC1 $\alpha$ or with AllStars negative control siRNA at a final concentration of 30 $\mathrm{nmol} / \mathrm{L}$, using Lipofectamine 2000 reagent (Life Technologies, Grand Island, NY), according to the manufacturer's instructions.

\section{RNA-Seq Analysis}

Samples were sequenced at the Cornell University Transcriptional Regulation and Expression Facility (Ithaca, NY; zebrafish melanoma model) and the New York University Genome Technology Core (New York, NY) WM3248 human melanoma cells) using Hi-Seq. Per-read per-sample FASTQ files were generated using bcl2fastq2 Conversion software version 2.20 (Illumina, San Diego, CA) to convert per-cycle BCL base call file output by the sequencing instrument into the FASTQ format. Alignment software, STAR version 2.6.1d ${ }^{15}$ was used for mapping reads to the hg19 human and Zv9 zebrafish reference genomes, respectively, and Fastq Screen software version 0.13.0 (Barbraham Institute, Cambridge, UK) was utilized to check for sample contamination. FeatureCounts software ${ }^{16}$ Subread package version 1.6.3 was used to generate matrices of read counts for annotated genomic features of each human and zebrafish data set. For differential gene statistical comparisons between groups of samples, the DESeq2 package ${ }^{17}$ Bioconductor version 3.10 in the $\mathrm{R}$ statistical programming environment was utilized. Genes with a false-discovery rate of $<0.05$ were considered significantly different between groups. Heatmaps were generated using the pheatmap $\mathrm{R}$ software package version 1.0.12 (Raivo Kolde, University of Tartu, Estonia). Pathway analysis was performed using ClusterProfiler version 3.14.3 $3^{18}$ to identify enriched Kyoto Encyclopedia of Genes and Genomes signaling pathways based on top significantly expressed genes. Gene Set Enrichment Analysis in Python version 3.8 was used to identify genes that were enriched in a specific phenotype of interest. Additional gene list enrichment analysis of PGC1 $\alpha-$ regulated transcripts was performed using the EnrichR web server (https://amp.pharm.mssm.edu/Enrichr, last accessed December 17, 2019). ${ }^{19}$ RNA-Seq data were deposited at the NCBI Gene Expression Omnibus public repository (https://www.ncbi.nlm.nih.gov/geo, accession numbers GSE144117 and GSE145101).

\section{Oxygen Consumption Rate Assay}

Oxygen consumption rate (OCR) of human primary melanoma cell lines was assessed using the OCR Assay Kit (Cayman Chemicals, Ann Arbor, MI), according to the manufacturer's instructions. Antimycin A, a mitochondrial electron transport chain inhibitor, was used as a negative control for zero oxygen consumption. Fluorescence was measured using a SpectraMax M3 plate reader (Molecular Devices, LLC, San Jose, CA) at $380 \mathrm{~nm}$ (excitation) and 650 $\mathrm{nm}$ (emission). Oxygen consumption was calculated using linear regression to determine the slope for each signal profile.

\section{Metabolic Profiling}

Metabolic phenotype profiling of 367 carbon energy and nitrogen substrates was performed in WM35 and WM3248 
primary melanoma cell lines using Phenotype MicroArrays M1 to M4 (Biolog, Hayward, CA), according to the manufacturer's instructions. Briefly, $2 \times 10^{4}$ cells were seeded in each well of four 96-well plates. After 48 hours of incubation at $37^{\circ} \mathrm{C}$ in $5 \% \mathrm{CO}_{2}$, Biolog Redox Dye $\mathrm{MA}$ (Biolog) was added to wells. Tetrazolium dye reduction for each well was determined using a SpectraMax M3 plate reader (Molecular Devices) at an absorbance of $590 \mathrm{~nm}$.

\section{Melanoma Patients and Tumor Tissue Specimens}

Patients with primary melanoma $(n=46)$ were enrolled in the New York University Langone Medical Center Interdisciplinary Melanoma Cooperative Group biospecimen database, with prospectively recorded demographic, clinical, and pathologic data. ${ }^{20}$ The study protocol was approved by the New York University School of Medicine institutional review board. All patients gave written, informed consent before the study.

\section{PGC1 $\alpha$, Ki-67, and SCARA3 IHC}

Immunohistochemistry (IHC) was performed on formalinfixed paraffin-embedded primary melanoma specimens, as described. ${ }^{21}$ Antibodies used were anti-PGC1 $\alpha$ rabbit monoclonal antibody (catalog number ab84139, 1:200 dilution; Abcam, Cambridge, MA), anti-Ki-67 rabbit monoclonal antibody D2H10 (catalog number 9207, 1:800 dilution; Cell Signaling Technology, Danvers, MA), or anti-scavenger receptor class A, member 3 (SCARA3) rabbit polyclonal N2C2 (catalog number GTX100595, 1:500 dilution; GeneTex, Irvine, CA). PGC1 $\alpha$ expression was scored based on staining intensity and distribution in tumors $^{22}$ by an attending melanoma pathologist (F.D.) who was blinded to the clinical data. A score of 0 (absent), 1 (weak), or 2 (strong) was assigned to each sample based on PGC1 $\alpha$ staining intensity, compared to positive and negative control tissues. Next, the distribution of PGC1 $\alpha$ staining was assessed, in which focal staining (1\% to $50 \%$ of the tumor) was a score of 1 and diffuse staining ( $>50 \%$ of the tumor) was a score of 2 . The final PGC1 $\alpha$ expression score was the sum of intensity and distribution scores, resulting in three discrete PGC1 $\alpha$ expression groups: 0, absent; 2, weak; and 3, 4, strong. Ki-67 expression was scored as a percentage of positive tumor cells, as described. ${ }^{23}$

\section{RNA Extraction and Quantitative RT-PCR}

Total RNA was isolated from cells using a QIAshredder and RNeasy Plus Mini Kit (Qiagen), according to the manufacturer's instructions. For cDNA synthesis, $0.5 \mu \mathrm{g}$ of RNA was reverse-transcribed using a QuantiTect Reverse Transcription kit (Qiagen). Quantitative RT-PCR was performed using TaqMan gene expression assays (PPARGC1A, Hs00173304_m1; 18S, Hs03003631_g1), TaqMan Fast Advanced Master Mix, and the Applied Biosystems
StepOnePlus Real-Time PCR System (Life Technologies). All data were normalized to expression of the $18 S$ housekeeping gene. Relative PGC1 $\alpha$ expression levels between samples were calculated using the $2^{-\Delta \Delta \mathrm{Ct}}$ method. ${ }^{24}$

\section{Results}

\section{Up-Regulation of 0xidative Phosphorylation in Invasive Melanoma}

To identify mediators of primary melanoma invasion, we developed a zebrafish tumor model $[\operatorname{Tg}($ mitfa:B$R A F(\mathrm{~V} 600 \mathrm{E})) ; p 53(\mathrm{lf}) ; m i t f a(\mathrm{lf})]$ that allowed expression of constitutively active Myr-RSK1 in rescued melanocytes from a MiniCoopR tol2-based transposon vector. ${ }^{14}$ Previously, this model was used to show that activation of RSK1, a key downstream effector of ERK1/2 signaling, ${ }^{25}$ promotes vertical invasion of melanoma. ${ }^{26}$ To gain a more detailed understanding of the mechanisms underlying the invasive growth of primary melanoma, this investigation identified cellular processes that are altered between $\mathrm{Myr}$ RSK1 and Green fluorescent protein (control) tumors in this in vivo model using RNA-Seq (Figure 1A). The analysis revealed 1249 transcripts whose expression was significantly different (adjusted $P<0.05$ ) between Myr-RSK1 tumors and Green fluorescent protein control tumors (Figure 1B and Supplemental Table S1). Notably, analysis of differentially expressed genes between control and RSK1-activated tumors identified significant enrichment for gene ontology terms associated with metabolism, including electron transport chain, oxidative phosphorylation, and fatty acid biosynthesis (Figure 1C and Supplemental Tables S2 and S3). The analysis also identified enrichment of gene ontology terms that have been associated with melanoma development and progression (Figure 1C), including extracellular matrix reorganization, cell cycle progression, regulation of growth, calcium signaling, histone modifications, and RNA processing. ${ }^{27-30}$ Further analysis using Kyoto Encyclopedia of Genes and Genomes gene sets identified 51 genes belonging to the OXPHOS pathway that were up-regulated in Myr-RSK1 tumors relative to Green fluorescent protein control tumors (Figure 1D).

To establish a role for increased OXPHOS in invasive primary human melanoma, this study used a cell-based assay to assess the OCR in a panel of RGP and VGP human melanoma cell lines (WM35, WM278, WM115, and WM3248). In this assay, the signal from a phosphorescent oxygen probe was quenched by oxygen, resulting in a signal that was inversely proportional to the amount of oxygen present in the sample. The results showed that the OCR was significantly higher in more invasive WM3248 VGP melanoma cells compared to RGP and less invasive VGP melanoma cell lines (WM35, WM278, and WM115) (Figure 1E). Finally, to understand the utilization of carbon sources to fuel this increased OXPHOS, a metabolic 
A

Tg(mitfa:BRAF(V600E));p53(If);mitfa(If)

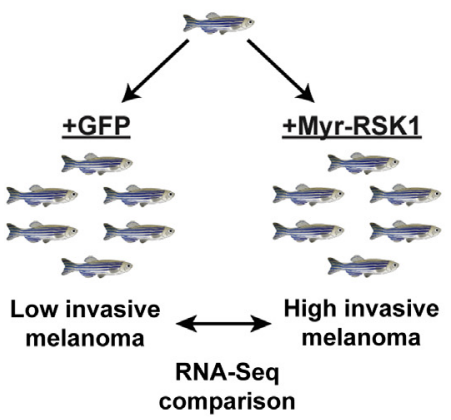

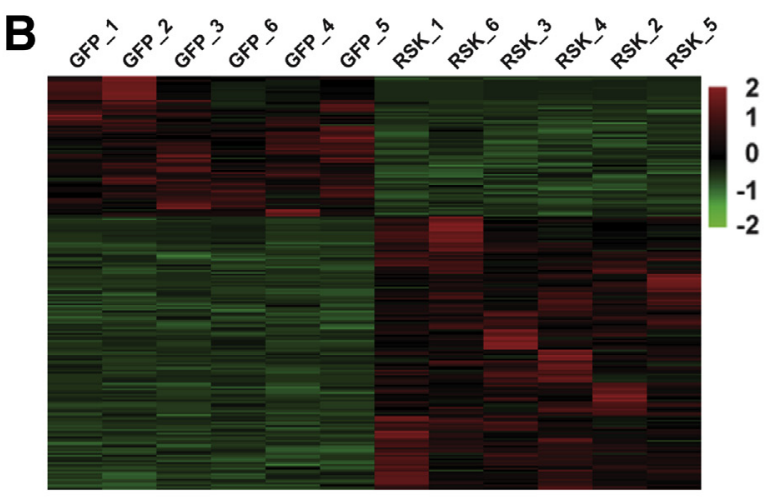

D

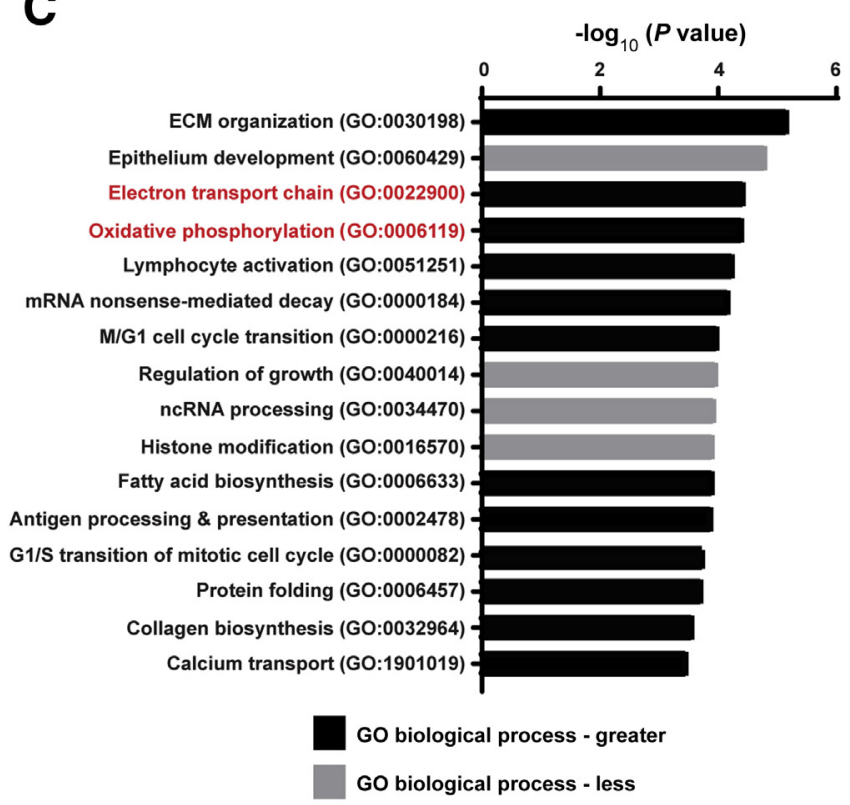

KEGG - Oxidative phosphorylation

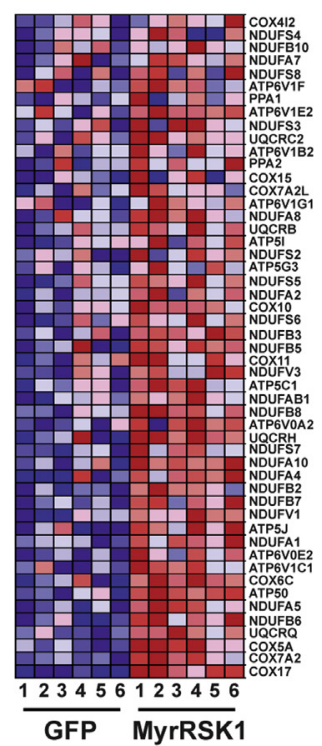

$\mathbf{E}$

Oxygen consumption rate

$\mathbf{F}$

Utilization of glutamine or arginine carbon sources
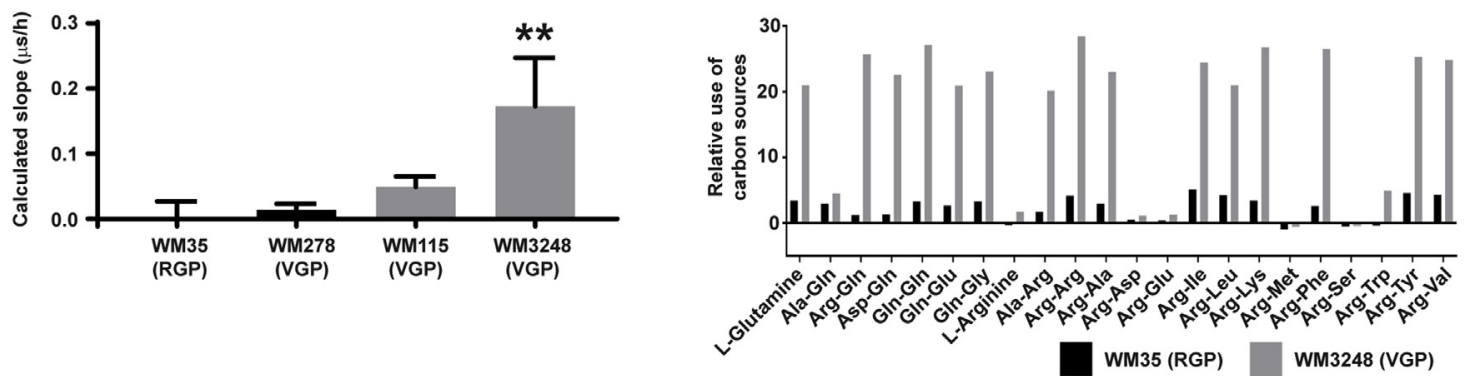

Figure 1 A zebrafish model identification of increased oxidative phosphorylation in invasive melanoma. A: Zebrafish melanoma model used for RNA-Seq comparison of low- and high-invasive melanoma. Six independent tumors from each experimental group [green fluorescent protein (GFP) control or constitutively active/myristoylated ribosomal protein S6 kinase (Myr-RSK)-1] were used for RNA-Seq comparative analysis. B: Heatmap showing 1249 genes differentially expressed between zebrafish melanomas expressing GFP (control) or Myr-RSK1. Red and green indicate up- and down-regulated genes, respectively. C: Gene ontology analysis showing enrichment of biological processes among genes differentially expressed between Myr-RSK1 and GFP data sets. D: Heatmap showing 51 genes from the oxidative phosphorylation Kyoto Encyclopedia of Genes and Genomes (KEGG) gene set that are significantly up-regulated in RSK1 tumors. Red and blue indicate up- and down-regulation, respectively. E: 0xygen consumption rate for radial growth phase (RGP) (WM35) and vertical growth phase (VGP) (WM278, WM115, and WM3248) melanoma cell lines of increasing invasive potential. F: Metabolic profiling assay comparing utilization of glutamine and arginine as carbon sources in RGP (WM35) versus VGP (WM3248) melanoma cells. Data are expressed as means \pm SD. $n=6$ per group (B). ${ }^{* *} P<0.01$ versus all other groups (one-way analysis of variance). ECM, extracellular matrix; G0, gene ontology; nc, noncoding. 

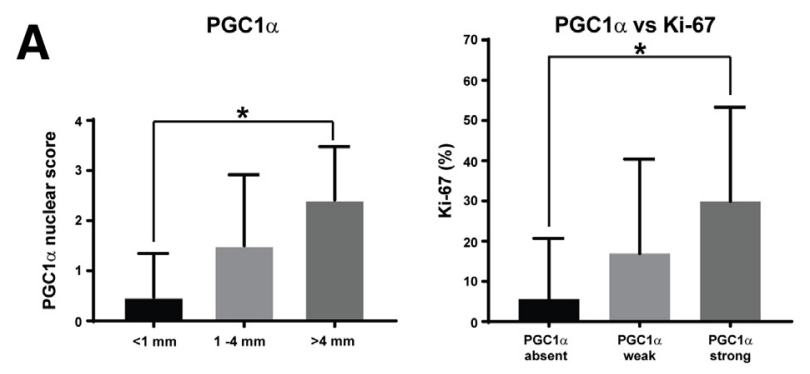

\section{B}
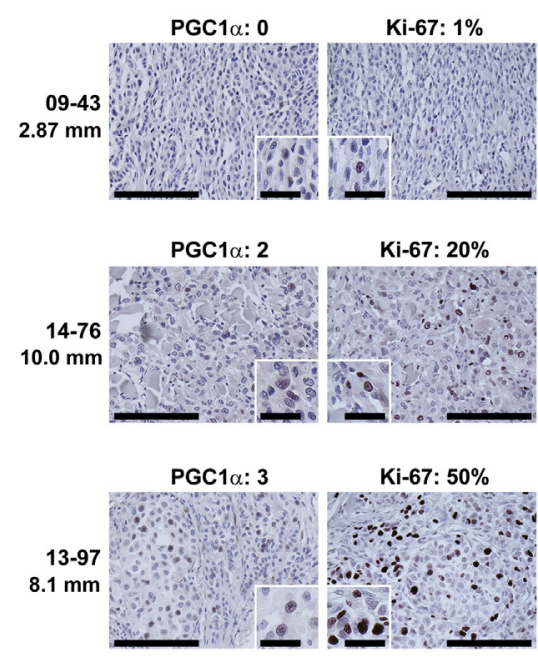

Figure 2 Associations between peroxisome proliferator-activated receptor $\gamma$ coactivator (PGC)-1 $\alpha$ and primary melanoma thickness and Ki-67 expression. A: Top panel: $\mathrm{PGC} 1 \alpha$ expression in primary melanomas of $4 \mathrm{~mm}$ thickness. Bottom panel: Ki-67 expression according to PGC1 $\alpha$ expression (absent, weak, or strong). B: Representative immunohistochemistry photomicrographs showing nuclear expression of PGC1 $\alpha$ (left column) and Ki67 (right column) in primary melanoma specimens, each of which is of the nodular melanoma histologic subtype. Insets show the main image at higher magnification. Data are expressed as means \pm SD. $n=46$ (A). ${ }^{*} P<0.05$ (two-tailed unpaired $t$-test). Scale bars: $0.2 \mathrm{~mm}$ (main images); $0.1 \mathrm{~mm}$ (insets). Original magnification: $\times 10$ (main images); $\times 40$ (insets).

phenotype microarray was used to compare RGP (WM35) and high invasive VGP (WM3248) melanoma cell lines. WM3248 cells showed increased utilization of glutamine and arginine carbon sources relative to WM35 cells (Figure 1F), suggesting that glutamine and arginine metabolism might support OXPHOS and an invasive VGP melanoma phenotype. Taken together, these results suggest a role for increased OXPHOS in primary melanoma invasion.

\section{Association between Expression of PGC $1 \alpha$ and Primary Melanoma Invasion and Proliferation}

The transcriptional coactivator $\mathrm{PGC} 1 \alpha$ promotes mitochondrial biogenesis in various tissues to increase energy production through OXPHOS. ${ }^{31}$ More recently, PGC1 $\alpha$ was shown to promote OXPHOS in metastatic melanoma. ${ }^{10,11}$ Based on the results of the OCR assays in RGP and VGP melanoma cell lines, which showed that OXPHOS was elevated in highly invasive cells in this study, it was hypothesized that PGC1 $\alpha$ expression would be positively associated with tumor thickness in primary melanoma specimens. To test this hypothesis, IHC was used to assess PGC1 $\alpha$ protein expression in a cohort of primary melanoma specimens $(n=46)$ (Supplemental Table S4). Nuclear expression of PGC1 $\alpha$ was determined using a composite scoring method that reflected staining intensity and distribution (Materials and Methods), and samples were stratified into groups using the following IHC scale: 0 , absent PGC1 $\alpha$ expression; 2, weak PGC1 $\alpha$ expression; or 3 or 4 , strong PGC1 $\alpha$ expression. The results showed that 18 of 46 tumors (39.1\%) had absent nuclear expression of PGC1 $\alpha, 12$ of 46 tumors $(26.1 \%)$ had weak nuclear expression of PGC1 $\alpha$, and 16 of 46 tumors (34.8\%) had strong nuclear expression of PGC1 $\alpha$. Nuclear expression of PGC1 $\alpha$ was significantly higher in thick, more invasive primary melanomas compared to thin, less invasive primary melanomas (Figure 2), and a significant positive correlation was found to exist between $\mathrm{PGC} 1 \alpha$ expression and tumor thickness (Pearson $r=0.437, P=0.0024)$. In parallel, the expression of the cellular proliferation marker Ki-67 was assessed by IHC in the same primary melanoma tissue cohort. A significant positive association between PGC1 $\alpha$ and Ki-67 expression was found (Figure 2). All tumors shown in Figure 2 belonged to the nodular melanoma histologic subtype (Supplemental Figure S1). These results are consistent with a role for PGC1 $\alpha$ in promoting the vertical growth and invasion that are a hallmark of early melanoma progression, at least in part through the up-regulation of OXPHOS.

Associations between PGC1 $\alpha$ Regulation of Expression of Genes and 0xidative Stress, Growth, and Invasion of Primary Melanoma Cells

This study next sought to elucidate how PGC1 $\alpha$ promotes primary melanoma progression. RNA interference was used to deplete PGC1 $\alpha$ levels in invasive WM3248 primary melanoma cells, and transfection with siRNA targeting PGC $1 \alpha$ produced a $>60 \%$ reduction in PGC $1 \alpha$ mRNA levels (Figure 3A). To identify transcripts regulated by PGC1 $\alpha$ in primary melanoma, RNA-Seq was performed using RNA extracted from WM3248 cells that were transfected with siRNA against PGC1 $\alpha$ or negative control siRNA (Figure 3B). Genes whose expression was significantly altered after PGC1 $\alpha$ knockdown (adjusted $P<0.05$ and $\log _{2}$ fold-change $\geq 0.5$ or $\leq-0.5$ ) (Supplemental Table S5) were analyzed for functional enrichment among canonical pathways (Wikipathways). In PGC1 $\alpha$-depleted WM3248 cells, this study identified up-regulation of oxidative stress, cell cycle control, DNA damage response, senescence and autophagy, and apoptosis; and downregulation of focal adhesion/phosphatidylinositol 3kinase-Akt signaling, matrix metalloproteinases, focal 
A

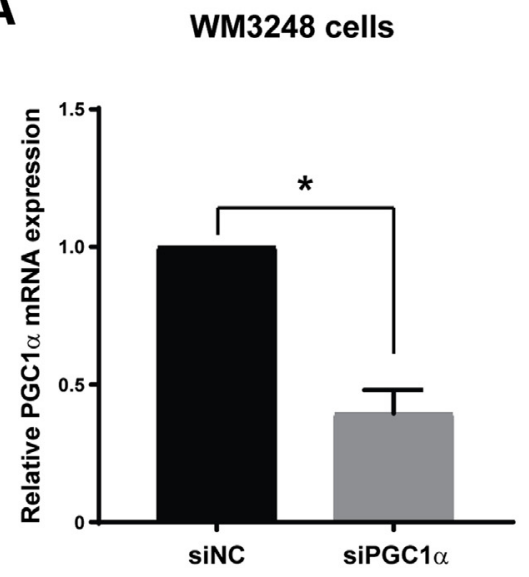

C

Wikipathways - upregulated with PGC1 $\alpha$ knockdown

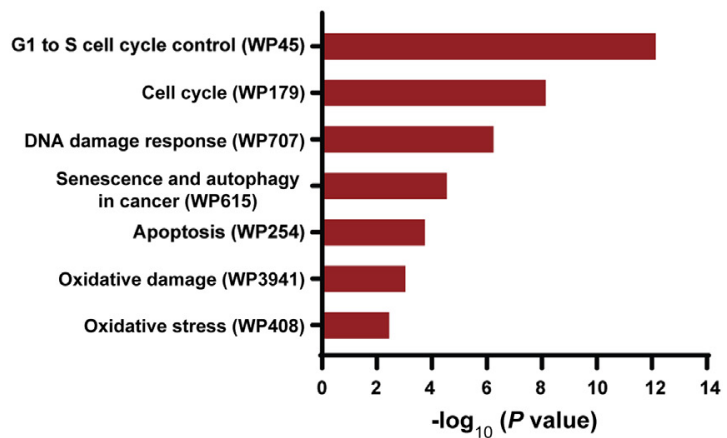

\section{B PGC1 $\alpha$ knockdown in WM3248 cells}

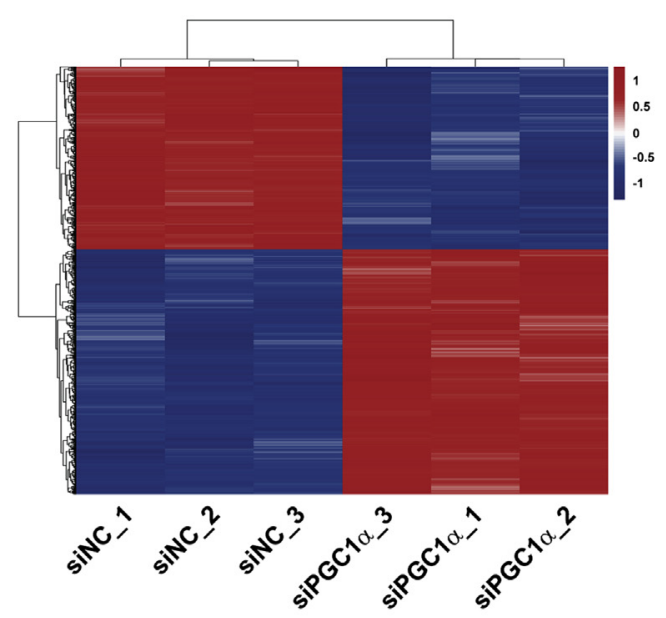

D

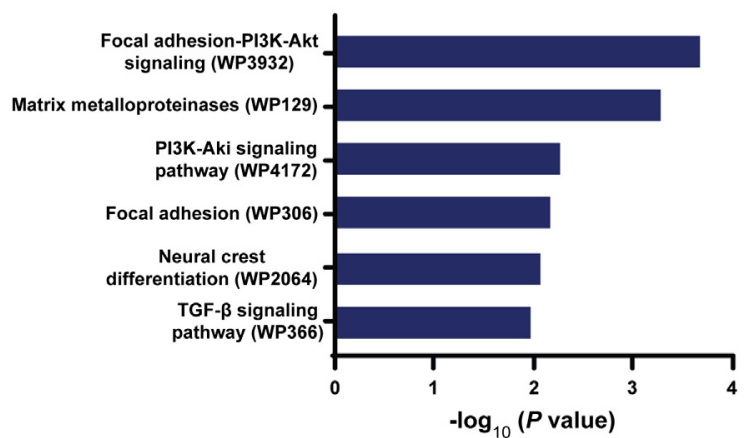

Figure 3 Peroxisome proliferator-activated receptor $\gamma$ coactivator (PGC)-1 $\alpha$ knockdown induction of transcriptional changes associated with oxidative stress, growth, and invasion in WM3248 primary melanoma cells. A: Knockdown of PGC1 $\alpha$ mRNA in WM3248 primary melanoma cells after transfection with siRNA against PGC1 $\alpha$ (siPGC1 $\alpha$ ) compared with negative control siRNA (siNC). B: Heatmap for comparison of gene expression (by RNA-Seq) between WM3248 cells transfected with siPGC1 $\alpha$ or siNC. Red and blue indicate up- and down-regulated genes, respectively. C and D: Analyses of functional pathways (Wikipathways) enriched for genes that are up-regulated (C) or down-regulated (D) by PGC1 $\alpha$ knockdown in WM3248 melanoma cells. Data are expressed as means $\pm \mathrm{SD}$. ${ }^{*} P<0.05$ (two-tailed unpaired $t$-test). PI3K, phosphatidylinositol 3-kinase; TGF, transforming growth factor.

adhesion, neural crest differentiation, and transforming growth factor $\beta$ signaling (Figure 3, C and D, and Supplemental Tables S6 and S7). The observation that PGC1 $\alpha$-regulated transcripts were enriched for genes involved in cell cycle control was interesting, given the positive association between PGC1 $\alpha$ and Ki-67 identified earlier in primary melanoma specimens (Figure 2).

To further substantiate the link between PGC1 $\alpha$, OXPHOS, and oxidative stress in invasive primary melanoma, this study assessed the expression of SCARA3, a protein that depletes reactive oxygen species (ROS) to protect cells from oxidative stress. ${ }^{32}$ First, significantly higher expression of scara3 in invasive, RSK1-activated zebrafish melanomas compared to control tumors was observed (Figure 4A). Next, SCARA3 protein expression by IHC was assessed in a subset of primary melanoma tissues $(n=22)$ that we had also stained for PGC1 $\alpha$. The results showed co-localization and a positive association between
PGC1 $\alpha$ and SCARA3 levels in serial tissue sections of primary melanoma (Figure 4B). Together, these findings suggest that the activation of SCARA3 expression could represent an antioxidant response in some tumors that helps to mitigate ROS production and support the invasive growth of PGC $1 \alpha$ high/OXPHOS high primary melanoma.

\section{Discussion}

Although the effects of somatic mutations and aberrant cell signaling pathways on the transition of primary melanoma from radial to vertical growth have been previously studied, ${ }^{1-5}$ there has been a limited understanding of the contribution of altered tumor metabolism to the invasive growth of primary melanoma. The current analyses using a zebrafish model of invasive melanoma and human primary melanoma cell lines and patient tissues support an important 
A

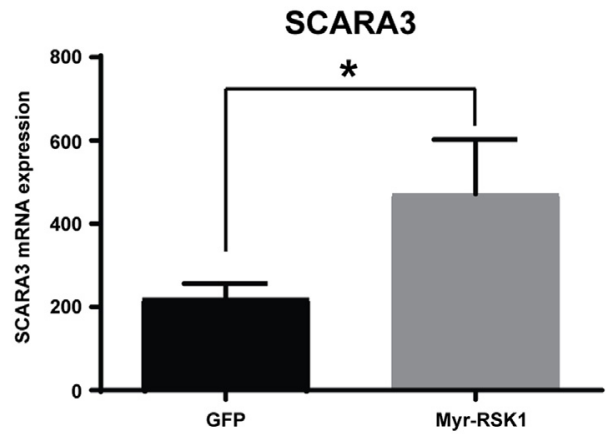

B

IHC: PGC1 $\alpha$
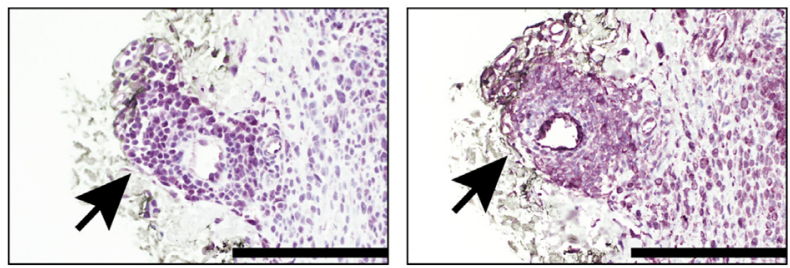

Figure 4 Co-expression of the reactive oxygen species scavenger, scavenger receptor class $A$ member 3 (SCARA3), with peroxisome proliferator-activated receptor $\gamma$ coactivator (PGC)-1 $\alpha$ in human primary melanoma specimens. A: Increased scara3 mRNA expression in tumors from myristoylated ribosomal protein S6 kinase (Myr-RSK1) zebrafish melanomas relative to green fluorescent protein (GFP) (control) zebrafish tumors. B: Representative immunohistochemistry (IHC) photomicrographs showing focal co-localization of PGC1 $\alpha$ (left panel) and SCARA3 (right panel) expression in serial tissue sections from human primary melanoma. Arrows denote areas of PGC1 $\alpha$ and SCARA3 positive staining. Data are expressed as means \pm SD. $n=22$ cases analyzed $(\mathbf{B}) .{ }^{\star} P<0.05$. Scale bars $=0.2 \mathrm{~mm}$. Original magnification, $\times 10$.

role for OXPHOS in primary melanoma invasive growth. These metabolic analyses identified increased OCR and utilization of glutamine by invasive primary melanoma cells. This study shows a role for PGC1 $\alpha$ in mediating this invasive phenotype, and that its protein expression is associated with increased primary melanoma thickness and $\mathrm{Ki}$ 67 expression. The suppression of PGC1 $\alpha$ expression in primary melanoma cells led to alterations in oxidative damage/stress, cell cycle control, focal adhesion, activity of matrix metalloproteinases, and transforming growth factor $\beta$ signaling, among other pathways. The links between PGC $1 \alpha$, OXPHOS, oxidative stress, and the progression of primary melanoma were further substantiated by an increased expression of the ROS scavenger scara3 in invasive zebrafish tumors and its colocalization with PGC1 $\alpha$ in human primary melanoma specimens, which is consistent with an antioxidant response in these tumors.

This study used a zebrafish model with a MiniCoopR expression system that produced melanocyte-specific activation of RSK1 and BRAF V600E, in addition to loss of function of p53 and MITF, to investigate the processes underlying invasive melanoma growth. ${ }^{14}$ The biological relevance of this model relates to the observation that RSK1 is hyperactivated in highly invasive VGP melanomas compared to less invasive RGP melanomas, and that RSK1 inhibition suppresses melanoma cell migration, invasion, and proliferation. ${ }^{26}$ Zebrafish melanoma models have been shown to consistently recapitulate the human disease, as zebrafish melanocytes are distributed throughout the basal layer of the epidermis in a fashion similar to that in human melanocytes, whereas melanocytes localize primarily to hair follicles in mice. ${ }^{33}$ Zebrafish models have been used to identify novel melanoma drivers and suppressors in the context of tumor initiation and progression, and to visualize interactions between melanoma and the tumor microenvironment. ${ }^{33}$ This study highlights the utility of this zebrafish model as a tool to study regulators of melanoma invasion. To substantiate these findings, parallel analyses of human primary melanoma cell lines and tumor specimens from melanoma patients were performed. Interestingly, the increased OXPHOS we observed in invasive RSK1-driven zebrafish melanomas was not accompanied by upregulated PGC1 $\alpha$ expression, which likely reflects underlying biological differences between humans and zebrafish. Rather than identifying homologous genes, the current zebrafish model revealed aberrant OXPHOS as a pathway that is associated with invasive growth of primary melanoma, and which could be interrogated in more detail in the context of human melanoma.

This study identified a role for altered tumor cell metabolism, and particularly for OXPHOS, in primary melanoma invasion. Previous studies have shown that some melanomas demonstrate the Warburg effect and are reliant on glycolysis for energy production, ${ }^{8}$ such as that induced by $B R A F$ activation, ${ }^{34}$ whereas other melanomas rely on oxidative phosphorylation associated with increased PGC1 $\alpha$ expression. ${ }^{11}$ Tumor cells can also utilize multiple energy sources, including pyruvate or fatty acids. ${ }^{35}$ Glutamine is an alternative carbon source for melanoma cells, and feeds the tricarboxylic acid cycle as well as driving fatty acid biosynthesis. ${ }^{36}$ In this context, glutamine-fueled mitochondrial OXPHOS is decoupled from glycolysis and promotes metabolic plasticity of tumor cells that is associated with metastasis and drug resistance. For example, the metabolic switch of some melanomas to OXPHOS has been linked to resistance to mitogen-activated protein kinase pathway inhibitors. ${ }^{9,37}$ Furthermore, a recent study showed that OXPHOS is enriched in melanoma brain metastases compared to extracranial metastases, and that growth of melanoma brain metastases could be suppressed by an OXPHOS inhibitor, IACS-010759. ${ }^{38}$ The potential utility of this strategy in melanoma patients has been questioned, as such inhibitors might be expected to block OXPHOS not only in tumor cells but also in anti-tumor T cells. However, inhibition of OXPHOS does not appear to block effector T-cell function, and instead has been shown to sensitize melanoma xenografts to immune checkpoint inhibitors, possibly in part by blocking the activity of myeloid-derived suppressor cells. ${ }^{39}$ 
A central finding of this study was that PGC1 $\alpha$ protein levels were associated with increased primary melanoma thickness, invasion, and proliferation, suggesting that PGC1 $\alpha$ is involved in metabolic rewiring to increase OXPHOS and ATP production and drive an invasive primary melanoma phenotype. This finding is supported by reports that high PGC1 $\alpha$ expression is associated with a worse prognosis in metastatic melanoma. ${ }^{10,11}$ In contrast, Luo et $\mathrm{al}^{40}$ found that $\mathrm{PGC} 1 \alpha$ knockdown promoted invasion of metastatic melanoma cell lines and that PGC1 $\alpha$ levels were inversely correlated with vertical growth in specimens from patients with primary melanoma. That finding suggests that the impact of PGC1 $\alpha$ knockdown may be context specific. Additionally, Luo et $\mathrm{al}^{40}$ identified reduced levels of PGCIA mRNA in VGP versus RGP melanoma microarray data sets, but did not verify this finding with PGC1 $\alpha$ protein. In contrast, the present study assessed, by IHC, PGC1 $\alpha$ protein levels in tissues from patients with primary melanoma, and $\mathrm{PGC} 1 \alpha$ protein levels are more likely to reflect the true functional impact of PGC1 $\alpha$ in early melanoma progression, particularly given reports that have highlighted discrepancies between mRNA and protein expression in vivo. ${ }^{41,42}$

A consequence of a metabolic switch to OXPHOS in some melanomas is the increased production of ROS, which promotes oxidative stress, mitochondrial release of cytochrome C, and apoptosis. ${ }^{43}$ Tumor cells with high levels of PGC1 $\alpha$ may thus be protected from ROS in a PGC $1 \alpha-$ dependent manner, ${ }^{11}$ since PGC1 $\alpha$ inhibition has been correlated with elevated ROS generation and a hypoxiainducible factor $1 \alpha$-mediated switch to glycolysis. ${ }^{44}$ Moreover, increased apoptosis of PGC1 $\alpha$-inhibited A375P melanoma cells can be reversed by treatment with antioxidants. ${ }^{11}$ These findings also suggest that SCARA3 may be an important component of the antioxidant response in melanoma cells as they adapt to OXPHOS to sustain their growth and invasion during early melanoma progression. Further studies are needed to fully define the mechanisms by which PGC1 $\alpha$ mitigates the oxidative stress associated with ROS generation in the context of primary melanoma growth and invasion.

In conclusion, the current study data using a zebrafish melanoma model, as well as human primary melanoma cell lines and tumor tissues, support a role for PGC1 $\alpha$ in mediating a metabolic switch to glutamine-driven OXPHOS during early melanoma progression. PGC1 $\alpha$ is up-regulated in thick primary melanomas and drives melanoma cell invasion and proliferation by modulating the expression of genes involved in oxidative stress, cell cycle control, DNA damage response, apoptosis, focal adhesion, matrix metalloproteinases, phosphatidylinositol 3-kinase-Akt, and transforming growth factor $\beta$ signaling. This observation that highly invasive primary melanoma cells utilize glutamine to a greater extent than less invasive primary melanoma cells suggests that they might also be vulnerable to targeting glutamine metabolism or uptake. Several different therapeutic strategies have been proposed to exploit this preference. ${ }^{45,46}$ Ultimately, an understanding of the role of metabolic rewiring in primary melanoma invasion and growth is likely to provide insight into the biology underlying melanoma progression, and might highlight novel vulnerabilities that could be targeted to treat advanced disease.

\section{Acknowledgments}

We thank Chloe Goldman for technical assistance; the New York University Genome Technology Center for expert library preparation, sequencing, and data analysis; and the High Performance Computing Core, Medical Center Information Technology group (New York University Langone Medical Center) for computing resources.

\section{Author Contributions}

A.S. identified human melanoma samples for analysis, contributed significantly to writing manuscript; A.J. performed data analysis and literature review; I.B. performed IHC and assisted with RNA extraction; A.I. performed manuscript preparation; F.D. reviewed IHC and scored tissues; Y.H. performed zebrafish model development; K.G. was the primary contributor to intellectual and written components of the manuscript, performed statistical analyses, and designed and conducted experiments; I.O., oversaw the melanoma biobank and performed manuscript preparation.

\section{Supplemental Data}

Supplemental material for this article can be found at http://doi.org/10.1016/j.ajpath.2020.01.012.

\section{References}

1. Kibbi N, Kluger H, Choi JN: Melanoma: clinical presentations. Cancer Treat Res 2016, 167:107-129

2. Thomas AJ, Erickson CA: The making of a melanocyte: the specification of melanoblasts from the neural crest. Pigment Cell Melanoma Res 2008, 21:598-610

3. Mobley AK, Braeuer RR, Kamiya T, Shoshan E, Bar-Eli M: Driving transcriptional regulators in melanoma metastasis. Cancer Metastasis Rev 2012, 31:621-632

4. Ryu B, Moriarty WF, Stine MJ, DeLuca A, Kim DS, Meeker AK, Grills LD, Switzer RA, Eller MS, Alani RM: Global analysis of BRAFV600E target genes in human melanocytes identifies matrix metalloproteinase-1 as a critical mediator of melanoma growth. J Invest Dermatol 2011, 131:1579-1583

5. Hanniford D, Segura MF, Zhong J, Philips E, Jirau-Serrano X, Darvishian F, Berman RS, Shapiro RL, Pavlick AC, Brown B, Osman I, Hernando E: Identification of metastasis-suppressive microRNAs in primary melanoma. J Natl Cancer Inst 2015, 107: dju494

6. Hanahan D, Weinberg RA: Hallmarks of cancer: the next generation. Cell 2011, 144:646-674 
7. Pavlova NN, Thompson CB: The emerging hallmarks of cancer metabolism. Cell Metab 2016, 23:27-47

8. Fischer GM, Vashisht Gopal YN, McQuade JL, Peng W, DeBerardinis RJ, Davies MA: Metabolic strategies of melanoma cells: mechanisms, interactions with the tumor microenvironment, and therapeutic implications. Pigment Cell Melanoma Res 2018, 31: $11-30$

9. Gopal YN, Rizos H, Chen G, Deng W, Frederick DT, Cooper ZA, Scolyer RA, Pupo G, Komurov K, Sehgal V, Zhang J, Patel L, Pereira CG, Broom BM, Mills GB, Ram P, Smith PD, Wargo JA, Long GV, Davies MA: Inhibition of $\mathrm{mTORC} 1 / 2$ overcomes resistance to MAPK pathway inhibitors mediated by PGC1alpha and oxidative phosphorylation in melanoma. Cancer Res 2014, 74:7037-7047

10. Haq R, Shoag J, Andreu-Perez P, Yokoyama S, Edelman H, Rowe GC, Frederick DT, Hurley AD, Nellore A, Kung AL, Wargo JA, Song JS, Fisher DE, Arany Z, Widlund HR: Oncogenic BRAF regulates oxidative metabolism via PGC1alpha and MITF. Cancer Cell 2013, 23:302-315

11. Vazquez F, Lim JH, Chim H, Bhalla K, Girnun G, Pierce K, Clish CB, Granter SR, Widlund HR, Spiegelman BM, Puigserver P: PGC1alpha expression defines a subset of human melanoma tumors with increased mitochondrial capacity and resistance to oxidative stress. Cancer Cell 2013, 23:287-301

12. Ceol CJ, Houvras Y, Jane-Valbuena J, Bilodeau S, Orlando DA, Battisti V, Fritsch L, Lin WM, Hollmann TJ, Ferre F, Bourque C, Burke CJ, Turner L, Uong A, Johnson LA, Beroukhim R, Mermel CH, Loda M, Ait-Si-Ali S, Garraway LA, Young RA, Zon LI: The histone methyltransferase SETDB1 is recurrently amplified in melanoma and accelerates its onset. Nature 2011, 471: $513-517$

13. Shimamura A, Ballif BA, Richards SA, Blenis J: Rsk1 mediates a MEK-MAP kinase cell survival signal. Curr Biol 2000, 10:127-135

14. Iyengar S, Houvras Y, Ceol CJ: Screening for melanoma modifiers using a zebrafish autochthonous tumor model. J Vis Exp 2012 e50086

15. Dobin A, Davis CA, Schlesinger F, Drenkow J, Zaleski C, Jha S, Batut P, Chaisson M, Gingeras TR: STAR: ultrafast universal RNAseq aligner. Bioinformatics 2013, 29:15-21

16. Liao Y, Smyth GK, Shi W: featureCounts: an efficient general purpose program for assigning sequence reads to genomic features. Bioinformatics 2014, 30:923-930

17. Love MI, Huber W, Anders S: Moderated estimation of fold change and dispersion for RNA-seq data with DESeq2. Genome Biol 2014, $15: 550$

18. Yu G, Wang LG, Han Y, He QY: clusterProfiler: an R package for comparing biological themes among gene clusters. OMICS 2012, 16: 284-287

19. Chen EY, Tan CM, Kou Y, Duan Q, Wang Z, Meirelles GV, Clark NR, Ma'ayan A: EnrichR: interactive and collaborative HTML5 gene list enrichment analysis tool. BMC Bioinformatics 2013, $14: 128$

20. Wich LG, Hamilton HK, Shapiro RL, Pavlick A, Berman RS, Polsky D, Goldberg JD, Hernando E, Manga P, Krogsgaard M, Kamino H, Darvishian F, Lee P, Orlow SJ, Ostrer H, Bhardwaj N, Osman I: Developing a multidisciplinary prospective melanoma biospecimen repository to advance translational research. Am J Transl Res 2009, 1:35-43

21. Scanlon P, Tian J, Zhong J, Silva I, Shapiro R, Pavlick A, Berman R, Osman I, Darvishian F: Enhanced immunohistochemical detection of neural infiltration in primary melanoma: is there a clinical value? Hum Pathol 2014, 45:1656-1663

22. McCarty KS Jr, Szabo E, Flowers JL, Cox EB, Leight GS, Miller L, Konrath J, Soper JT, Budwit DA, Creasman WT, Seigler HF, McCarty KS: Use of a monoclonal anti-estrogen receptor antibody in the immunohistochemical evaluation of human tumors. Cancer Res $1986,46: 4244 s-4248 s$
23. Robinson EM, Rosenbaum BE, Zhang Y, Rogers R, Tchack J, Berman RS, Darvishian F, Osman I, Shapiro RL, Shao Y, Polsky D: Association between Ki-67 expression and clinical outcomes among patients with clinically node-negative, thick primary melanoma who underwent nodal staging. J Surg Oncol 2018, 118:150-156

24. Livak KJ, Schmittgen TD: Analysis of relative gene expression data using real-time quantitative PCR and the 2(-Delta Delta $\mathrm{C}(\mathrm{T})$ ) method. Methods 2001, 25:402-408

25. Anjum R, Blenis J: The RSK family of kinases: emerging roles in cellular signalling. Nat Rev Mol Cell Biol 2008, 9:747-758

26. Salhi A, Farhadian JA, Giles KM, Vega-Saenz de Miera E, Silva IP, Bourque C, Yeh K, Chhangawala S, Wang J, Ye F, Zhang DY, Hernando-Monge E, Houvras Y, Osman I: RSK1 activation promotes invasion in nodular melanoma. Am J Pathol 2015, 185:704-716

27. Alonso SR, Ortiz P, Pollan M, Perez-Gomez B, Sanchez L, Acuna MJ, Pajares R, Martinez-Tello FJ, Hortelano CM, Piris MA, Rodriguez-Peralto JL: Progression in cutaneous malignant melanoma is associated with distinct expression profiles: a tissue microarraybased study. Am J Pathol 2004, 164:193-203

28. Hofmann UB, Houben R, Brocker EB, Becker JC: Role of matrix metalloproteinases in melanoma cell invasion. Biochimie 2005, 87: 307-314

29. Sarkar D, Leung EY, Baguley BC, Finlay GJ, Askarian-Amiri ME: Epigenetic regulation in human melanoma: past and future. Epigenetics 2015, 10:103-121

30. Sun J, Lin S, Keeley T, Yang S: Disseminating melanoma cells surf on calcium waves. Mol Cell Oncol 2015, 2:e1002714

31. Scarpulla RC: Metabolic control of mitochondrial biogenesis through the PGC-1 family regulatory network. Biochim Biophys Acta 2011, 1813:1269-1278

32. Han HJ, Tokino T, Nakamura Y: CSR, a scavenger receptor-like protein with a protective role against cellular damage caused by UV irradiation and oxidative stress. Hum Mol Genet 1998, 7: 1039-1046

33. van Rooijen E, Fazio M, Zon LI: From fish bowl to bedside: the power of zebrafish to unravel melanoma pathogenesis and discover new therapeutics. Pigment Cell Melanoma Res 2017, 30: 402-412

34. Ratnikov BI, Scott DA, Osterman AL, Smith JW, Ronai ZA: Metabolic rewiring in melanoma. Oncogene 2017, 36:147-157

35. Nakajima EC, Van Houten B: Metabolic symbiosis in cancer: refocusing the Warburg lens. Mol Carcinog 2013, 52:329-337

36. Filipp FV, Ratnikov B, De Ingeniis J, Smith JW, Osterman AL, Scott DA: Glutamine-fueled mitochondrial metabolism is decoupled from glycolysis in melanoma. Pigment Cell Melanoma Res 2012, 25: $732-739$

37. Zhang G, Frederick DT, Wu L, Wei Z, Krepler C, Srinivasan S, et al: Targeting mitochondrial biogenesis to overcome drug resistance to MAPK inhibitors. J Clin Invest 2016, 126:1834-1856

38. Fischer GM, Jalali A, Kircher DA, Lee WC, McQuade JL, Haydu LE, Joon AY, Reuben A, de Macedo MP, Carapeto FCL, Yang C, Srivastava A, Ambati CR, Sreekumar A, Hudgens CW, Knighton B, Deng W, Ferguson SD, Tawbi HA, Glitza IC, Gershenwald JE, Vashisht Gopal YN, Hwu P, Huse JT, Wargo JA, Futreal PA, Putluri N, Lazar AJ, DeBerardinis RJ, Marszalek JR, Zhang J, Holmen SL, Tetzlaff MT, Davies MA: Molecular profiling reveals unique immune and metabolic features of melanoma brain metastases. Cancer Discov 2019, 9:628-645

39. Kim SH, Li M, Trousil S, Zhang Y, Pasca di Magliano M, Swanson KD, Zheng B: Phenformin inhibits myeloid-derived suppressor cells and enhances the anti-tumor activity of PD-1 blockade in melanoma. J Invest Dermatol 2017, 137:1740-1748

40. Luo C, Lim JH, Lee Y, Granter SR, Thomas A, Vazquez F, Widlund HR, Puigserver P: A PGClalpha-mediated transcriptional axis suppresses melanoma metastasis. Nature 2016, 537: $422-426$ 
41. Tian Q, Stepaniants SB, Mao M, Weng L, Feetham MC, Doyle MJ, Yi EC, Dai H, Thorsson V, Eng J, Goodlett D, Berger JP, Gunter B, Linseley PS, Stoughton RB, Aebersold R, Collins SJ, Hanlon WA, Hood LE: Integrated genomic and proteomic analyses of gene expression in mammalian cells. Mol Cell Proteomics 2004, 3:960-969

42. Vogel C, Abreu Rde S, Ko D, Le SY, Shapiro BA, Burns SC, Sandhu D, Boutz DR, Marcotte EM, Penalva LO: Sequence signatures and mRNA concentration can explain two-thirds of protein abundance variation in a human cell line. Mol Syst Biol 2010, 6:400

43. Wen S, Zhu D, Huang P: Targeting cancer cell mitochondria as a therapeutic approach. Future Med Chem 2013, 5:53-67
44. Lim JH, Luo C, Vazquez F, Puigserver P: Targeting mitochondrial oxidative metabolism in melanoma causes metabolic compensation through glucose and glutamine utilization. Cancer Res 2014, 74: $3535-3545$

45. Qin JZ, Xin H, Nickoloff BJ: Targeting glutamine metabolism sensitizes melanoma cells to TRAIL-induced death. Biochem Biophys Res Commun 2010, 398:146-152

46. Wang Q, Beaumont KA, Otte NJ, Font J, Bailey CG, van Geldermalsen M, Sharp DM, Tiffen JC, Ryan RM, Jormakka M, Haass NK, Rasko JE, Holst J: Targeting glutamine transport to suppress melanoma cell growth. Int J Cancer 2014, 135:1060-1071 